\title{
High resolution measurements with miniature neutron scintillators in the SUR-100 zero power reactor
}

\author{
Edoardo L. BRUNETTO ${ }^{1,2}$, Fanny VITULLO ${ }^{1}$, Vincent LAMIRAND ${ }^{1,3}$, Klemen AMBROŽIČ ${ }^{1}$, \\ Daniel GODAT ${ }^{1}$, Michael BUCK ${ }^{4}$, Georg POHLNER ${ }^{4}$, Jörg STARFLINGER ${ }^{4}$, Andreas PAUTZ ${ }^{1,3}$ \\ ${ }^{1}$ École polytechnique fédérale de Lausanne (EPFL), Switzerland \\ ${ }^{2}$ Politecnico di Torino, Italy \\ ${ }^{3}$ Paul Scherrer Institut (PSI), Switzerland \\ ${ }^{4}$ University of Stuttgart, Germany
}

Corresponding author: vincent.lamirand@epfl.ch

\begin{abstract}
Three 1-mm $\mathrm{mm}^{3}$ miniature fiber-coupled scintillators have been used to perform $\mathrm{cm}$-wise resolution measurements of the thermal neutron flux within experimental channels of the SUR-100 facility, a zero power thermal reactor operated by the Institute of Nuclear Technology and Energy Systems at the University of Stuttgart. The detection system is developed at the École Polytechnique Fédérale de Lausanne in collaboration with the Paul Scherrer Institut. Thermal neutrons count rates were measured along the experimental channels I and II, which cross the reactor at the center and tangentially to the core, respectively. The reactor was modelled with the Monte Carlo neutron transport code Serpent-2.1.31. The comparison of experimental and computed reaction rate distributions showed a good agreement within the core region, with discrepancies within $2 \sigma$. An unexpected discrepancy, probably caused by a geometric inconsistency in the computational model of the reactor, was observed in the reflector region of the experimental channel I, where a $20 \%$ difference (i.e. 8\%) was found between experimental and simulated results. Significant discrepancies, respectively worth $10 \sigma$ and $15 \sigma$, were noticed at distance, in the lead shielding region, for both experimental channels I and II. In addition, reaction rate gradients across the $2.6 \mathrm{~cm}$ and $5.4 \mathrm{~cm}$ diameters of both channels were measured. A horizontal reaction rate gradient of $(9.09 \pm 0.20) \%$ was measured within $2.4 \mathrm{~cm}$ across the diameter of the experimental channel II, with a difference from computed results of $2 \%$. The absence of a vertical reaction rate gradient inside the experimental channel I was confirmed by measurements.
\end{abstract}

Keywords - Scintillation detectors, Monte Carlo simulations, Reaction rate profiles.

\section{INTRODUCTION}

$\mathrm{T}_{\mathrm{p}}^{\mathrm{H}}$ HE objective of the campaign presented hereafter was to perform high spatial resolution measurements to characterize the neutron thermal flux distribution inside experimental channels of the SUR-100 reactor, and to compare the measurement results with Monte Carlo simulations. The study benefited of the innovative miniature detector developed by the Laboratory for Reactor Physics and Systems Behaviour (LRS) at EPFL in collaboration with the Paul Scherrer Institut (PSI) [1]. Three units of the fiber-coupled scintillator detector were used in different arrangements to perform the flux characterization of the experimental channels of the reactor. Their small size results in a neutron flux perturbation lower than any other conventional detector and allows for measurements with high spatial discretization. All the profile measurements presented in this paper are given in relative reaction rates units. In addition, a preexisting MCNP [2] model of the reactor has been converted into Serpent2 [3] and used to run particle transport simulations. Monte Carlo simulations have been performed both to design the experiments, and to compare their results to the acquired measurements. The experimental campaign is described in the first section, followed by the modelling of the reactor, and finally the comparison and discussion of experimental and simulation results are presented.

\section{EXPERIMENTAL CAMPAIGN}

\section{A. The SUR-100 reactor}

The Siemens Training Reactor SUR-100 (in Fig. 1) is a thermal zero-power reactor built in 1964 and located in the University of Stuttgart. It is used for mainly for educational purposes by the Institut für Kernenergetik und Energiesysteme (IKE). The reactor has a maximum thermal power output of 1 $\mathrm{W}$, with a nominal power of $100 \mathrm{~mW}$. It is equipped with a homogeneous core, composed of solid cylindrical slabs of moderator with fuel particles dispersed inside. The core is provided with a lifting mechanism which can move axially the lower core section away from the upper one. The core halves are separated for the shutdown and brought together for the startup of the reactor. The reactivity regulation is performed with two cadmium control plates, which are steered into the core from below. The reactor is provided with various 
experimental channels, which allow the insertion of nuclear instrumentation into the core or in its proximity.

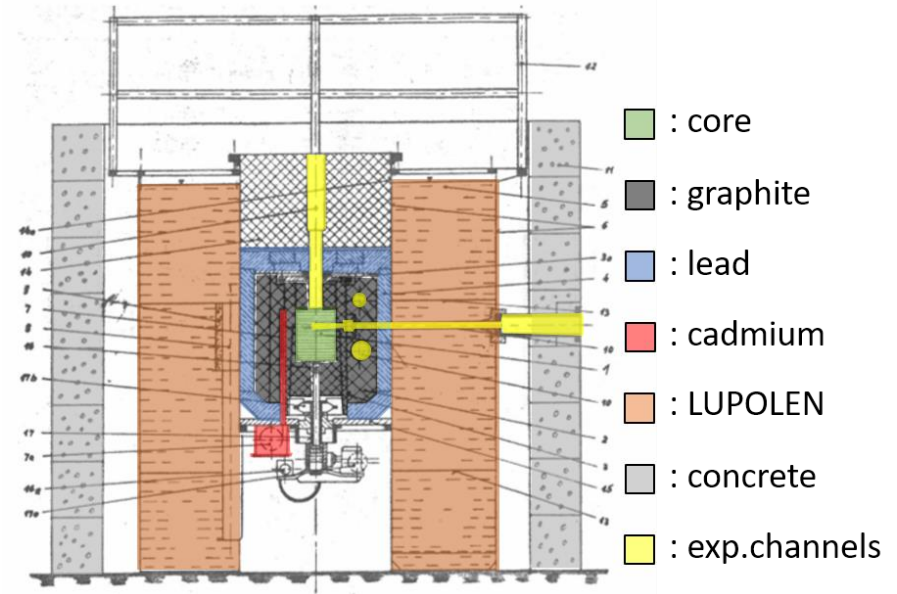

Fig. 1 - Scheme of the SUR-100 reactor

\section{B. Miniature neutron scintillator}

The miniature fiber-coupled neutron scintillator used in this study has been developed at the LRS-EPFL in collaboration with the Paul Scherrer Institut (PSI) [1]. Its miniature dimensions allow for highly localized measurements, while showing a good neutron sensitivity. Indeed, past experiments demonstrated improved performances with respect to others incore detecting devices, e.g. by showing a sensitivity one order of magnitude higher than a miniature fission chamber or a sCVD diamond detector [4]. The neutron sensitive area of the detector is $\mathrm{a} \sim 1 \mathrm{~mm}^{2}$ scintillator screen of zinc sulphide doped with ${ }^{6} \mathrm{Li}$ having a thickness of $0.28 \mathrm{~mm}$ [5]. The conversion of detected neutrons in charged particles is performed by the $(n, \alpha)$ reaction of ${ }^{6} \mathrm{Li}$. The alpha and triton particles arising from the reaction induce scintillation of the $\mathrm{ZnS}$ molecules of the crystal. Through this two-step process, the incoming neutron radiation is converted to a light signal. A plastic optical fiber [6] is coupled to the scintillator screen, guiding the scintillation photons to the read-out electronics. These consists of a SiPM for photon detection, and in the present study of a standard analog detection chain setup, depicted in Fig. 2. As a preliminary step to the experimental campaign in the SUR-100 reactor, the instrumentation was previously tested in the CROCUS zero power reactor of EPFL.

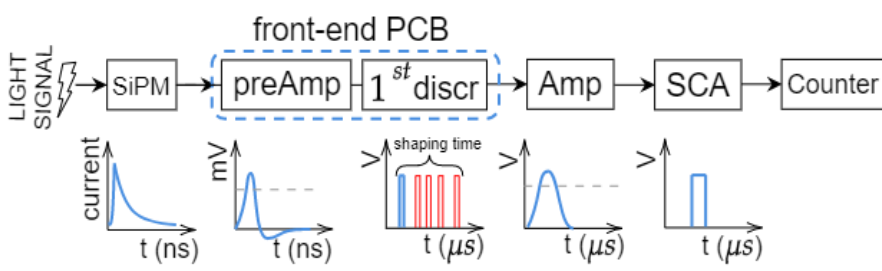

Fig. 2 - Standard analog detection chain.

\section{Detector arrangement}

The experimental instrumentation consisted in a multiple acquisition neutron detection system: three fiber-coupled scintillators have been used as detectors, with minor modifications with respect to the initial design of past studies [4]. The main improvement regards the aluminum cap, which has been reshaped in a new two-diameter configuration to improve the coupling with the fiber. An aluminum holder housing the three detectors tips was manufactured at a diameter of $26 \mathrm{~mm}$ corresponding to the internal diameter of the experimental channel I. Given the difference in diameter of the experimental channels, the diameter of the holding tube had the possibility to be adapted to a larger diameter $(54 \mathrm{~mm})$ by means of aluminum spacer rings. To move the detectors along the experimental channels, the front-end holder was mechanically coupled to an aluminum tube manufactured by the IKE laboratory, which was graduated to select the detectors' position inside the channels. The tube's handle allowed to adjust the angular orientation of the detectors inside the experimental channels. The choice of aluminum for the holding equipment was straightforward, given its small influence on thermal neutron flux, its availability, its low neutron absorption cross section and rapid decay of the induced activity.
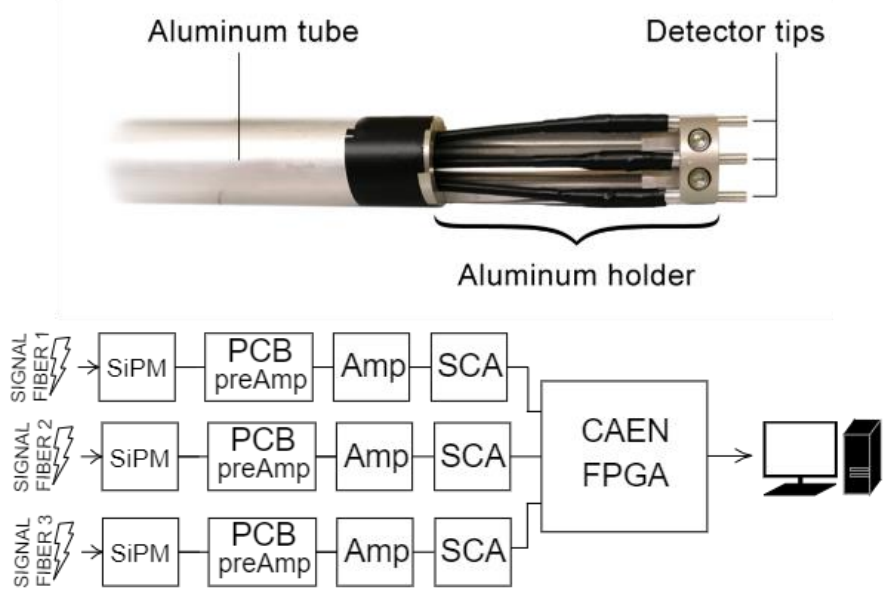

Fig. 3 - Fibers holder for measurements in SUR-100 (top) and read-out detection chain (bottom).

\section{Conducted experiments}

Steady-state and dynamic measurements were carried over along the campaign. We focus in the present article on the thermal flux characterization through the use of the miniature detectors, namely the measurements of thermal neutron flux profiles:

- along the central experimental channel I: $[-40,+40] \mathrm{cm}$ around core center,

- $\quad$ along the tangential channel II: $[-8,+46] \mathrm{cm}$ around core center,

- $\quad$ across the diameter of both channels I (vertically) and II (horizontally).

An explanatory scheme of the performed measurements is presented in Fig. 4. Depending on the experiment, the tube was 
slid into the channels with one of the possible configurations shown in Fig. 5.

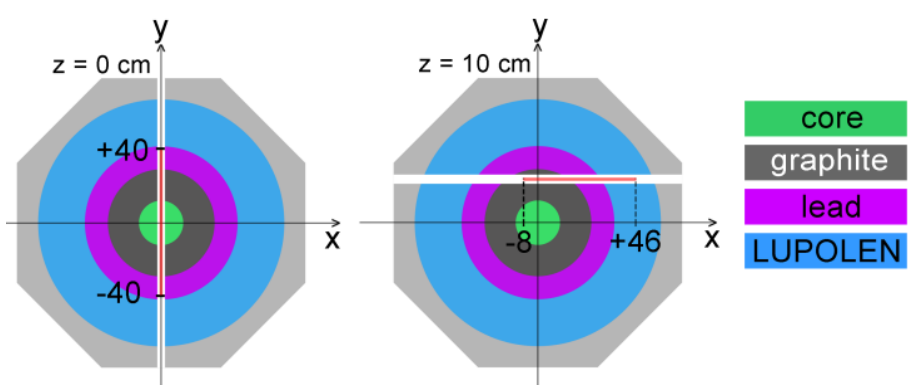

Fig. 4 - Simplified schemes of the profile measurements inside channel I (left) and channel II (right). The red lines indicate the spatial range in which measurements are performed.

The number of the detectors used was varied in dependence of the needs of each experiment. In most of the experiments, one of the miniature detectors (fiber 2) was removed from the holder and used for power monitoring. Count rates were recorded at each position for the time necessary to have statistical errors in the order of $0.1 \%$.

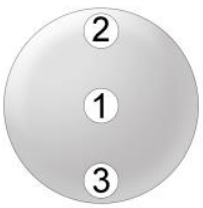

(a)

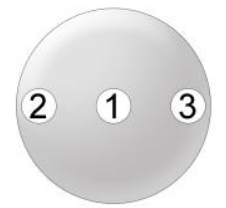

(b)

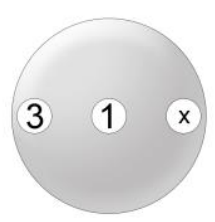

(c)
Fig. 5 - Different positioning of the detectors inside the experimental channels of SUR-100.

An additional measurement of the thermal flux profile inside the central channel was obtained with a neutron activation experiment which is conducted on a regular basis for teaching purposes at the SUR-100 reactor. The flux profile was retrieved by measuring with a High-Purity Germanium (HPGe) semiconductor detector the gamma-radiation emitted by some manganese samples irradiated in the reactor central channel for about 45 minutes. A cylindrical holder with the same diameter than the experimental channel was used to position 16 samples of ${ }^{55} \mathrm{Mn}$ inside the reactor core. The samples, consisting in tiny cylindrical rods of equal diameter with a length varying from 16.6 to $18.0 \mathrm{~mm}$, were positioned in the $2 \mathrm{~cm}$ spaced housings of the holder. The stable ${ }^{55} \mathrm{Mn}$ isotopes interact with thermal neutrons through the ${ }^{55} \mathrm{Mn}(\mathrm{n}, \gamma)$ neutron capture reaction, leading to the measurable decay of ${ }^{56} \mathrm{Mn}$ nuclei.

\section{SERPENT 2 MODEL OF THE SUR-100 REACTOR}

The Serpent 2 model hereafter presented results from the conversion of an already existent MCNP input: all the geometrical and materials details of the reactor have been taken from this reference, thus the same assumptions are considered. The main simplifications of the computational model concern:

- the absence of all the drive mechanisms, as they are not impacting the neutron economy relevantly;

- the absence of the experimental channel $10 / \mathrm{V}$, being it filled with graphite and therefore considered part of the reflector;

- the absence of the detector housings and the detectors themselves, since deemed non-impacting for the studies performed in this work because of their distance from the experimental channels;

- the absence of the aluminum coating of control plates, since they are assumed to not perturb the neutron flux in the thermal range of interest.

To improve its usability and its level of accuracy, the computational model has been adapted with minor modifications beyond its bare conversion, concerning shielding and control systems.

Despite its absence in the model provided by IKE, the concrete shielding has been modeled in Serpent2. Although the presence of the outermost layer of the shielding might not affect the neutronics in the center of the core, it is key for possible radiation protection studies e.g estimation of dose rate delivered to the operators. While the geometric dimensions of the concrete shielding have been taken from the official documentation from Siemens [7], its composition has been assumed "regular concrete" from the MCNP material compendium [8]. The concrete shielding has been shaped with a cylindrical shape, being this a simplification motivated by the absence of geometrical data on the real octagonal shape of the component at the time of the modeling procedure.

In addition, and to facilitate the modeling task of moving the control plates and the lower core section in different positions, the model is provided with a dedicated cell for each of them. This, together with the use of the translation command of Serpent2, enables to easily displace the elements without the need of a significant revision of the surfaces definition and opens to the use of apposite scripts to automatize the calculations. Thanks to these enhancements, the two control plates can be steered independently in the range $[0,-250] \mathrm{mm}$ and the lower core section (together with the lower section of the inner reflector) can be moved within the interval $[0,-45]$ $\mathrm{mm}$.

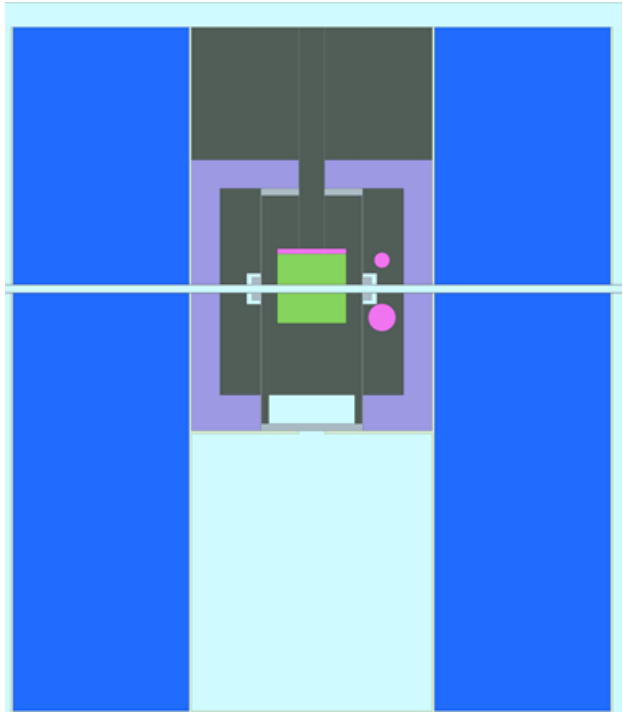

Fig. 6 - Vertical cut view of the Serpent2 SUR-100 model. 


\section{RESULTS}

\section{A. Neutron thermal flux profile along central channel I}

For this experiment, the miniature detectors 1 and 3 were moved along the length of channel I through the aluminum tube in the configuration (c) of Fig. 5, while the detector 2 was used as a monitor at a fixed position along the whole duration of the measurements in the tangential channel II. The results of the measurements recorded using detector 1 are depicted in Fig. 7. The count rates are normalized twice: first by detector 2 , then by the maximum measured value. The profile is symmetric with respect to the core center, given the overall agreement of the data measured on each side of the core. A slight difference is found to exist between the points lying in the interval from 20 to $28 \mathrm{~cm}$ far from the core center, with a disagreement in the order of $10 \%$.
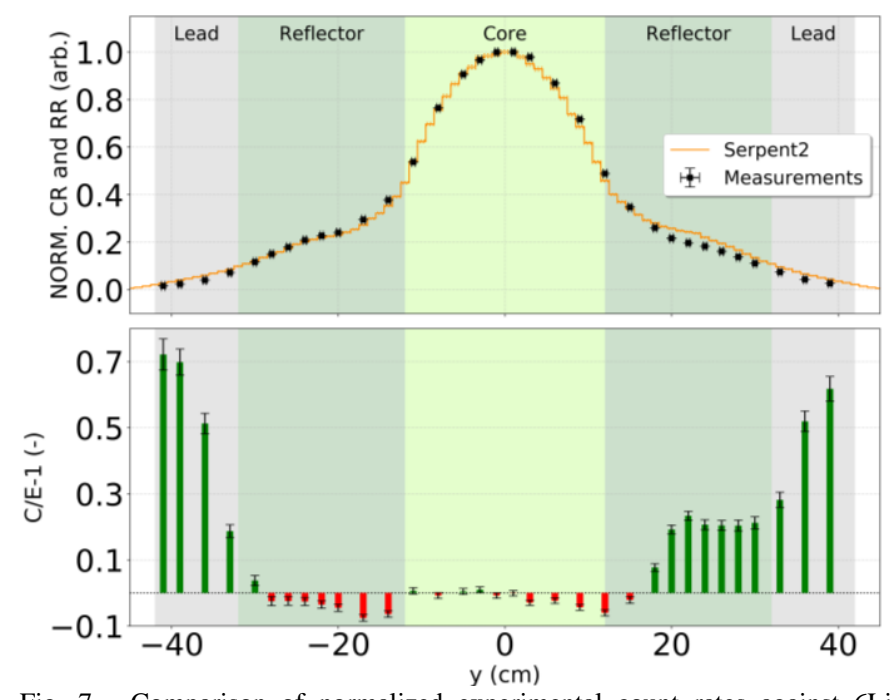

Fig. 7 - Comparison of normalized experimental count rates against 6L reaction rates predicted by simulations along experimental channel I (top) and observed discrepancies (bottom).

The measured data are then compared with computational results obtained through the Serpent 2 model. The real configuration of the reactor (i.e. power level, control plates position and filling of experimental channels) of the day of the measurement is implemented in the computational model, in order to reproduce the experiments as faithfully as possible. Cylindrical virtual detectors with the same radius of the channel $(26 \mathrm{~mm})$ and a thickness of $1 \mathrm{~cm}$ are used to score the neutrons reaction rate with the Lithium- 6 of the scintillator in every $\mathrm{cm}$ of the experimental channel in the range $[-45 ; 45] \mathrm{cm}$. The modeling of real-size detectors, given the miniature dimensions of the scintillators, was deemed to be unnecessary for the scope of the work at hand. Indeed, simulating real-size scintillator would cause a significant increase in the computational cost of the simulations, given the lower occurrence of neutrons interaction with a much smaller volume. Despite this, the smearing of the detectors is expected to introduce a discrepancy with the measured data, since the Monte Carlo results are in this way averaged on larger volumes. A depiction of the comparison of experimental and simulated data is also given in Fig. 7, as well as residuals.
The experimental results are seen to be particularly in agreement with the simulated data in the spatial range $[-30,18] \mathrm{cm}$, with a $\mathrm{C} / \mathrm{E}-1$ value lower than $10 \%$. It is worth noting that this overall good agreement proves the negligible neutron flux perturbation induced by the miniature detectors, not modeled for the computational simulation.

An asymmetry in the radial profile is found in the zone of the graphite reflector between +20 and $+30 \mathrm{~cm}$ away from the core, and it is not foreseen by the simulations. This discrepancy, which causes a disagreement of about $20 \%$, could be caused by localized change in material composition or some geometric asymmetry not considered in the computational model. Further away from the core center, a higher discrepancy is found to exist symmetrically from 30 to $40 \mathrm{~cm}$, with a C/E-1 value that reaches about $70 \%$. Given the presence of the lead shielding in the zone of highest disagreement, such a discrepancy could be motivated with the uncertainties in material composition or in its cross section. Since the data series are normalized for their maximum values (which is located in the core center), the disagreement observed could not necessarily be attributed to the peripheral zones and therefore at the lead shielding region. Nevertheless, the main suspicions fall on the lead shielding area, as it is mechanically and chemically less known than the core center region.

The results are compared as well with the activation experiment in Fig. 8 (top), where a substantial disagreement is found between the fiber measurements and the count rates retrieved with the activation technique.

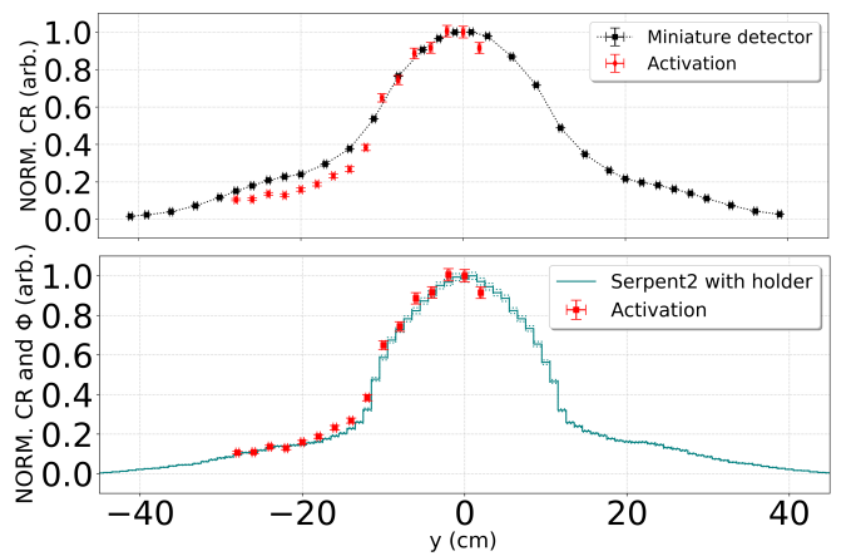

Fig. 8 - Direct comparison of the activation profile with measurements of miniature detector (top); Comparison of the activation profile with Serpent2 computed results (bottom).

The two measured neutron flux distributions differ particularly in the region outside the core, where a discrepancy of about $30 \%$ is observed. The reason behind this relevant discrepancy is found to be due to the presence of the plasticgraphite holder. The use of the activation holder causes a perturbation in the shape of the neutron flux distribution due to its constituent materials, which increase the thermal neutron flux in the core center. Indeed, the presence of PMMA enhance the neutron moderation while the graphite promotes the neutrons reflection towards the inner core region. Therefore, if the two flux distributions are plotted by normalizing the measurements for their respective maximum value (as in Fig. 8 
top), a meaningless comparison is obtained, with differences up to $50 \%$. A fair comparison between the activation measurements and the Serpent 2 results are performed by including in the computational model the activation holder. The comparison is shown in in Fig. 8 (bottom), where a good agreement is found between experimental and computational data, with C/E-1 values lower than $20 \%$.

\section{B. Neutron thermal flux profile along channel II}

Similarly to the central channel I case above, the upper tangential channel was deployed to characterize the thermal neutron flux distribution along its length. No experimental data on the flux distribution was available beforehand, due to the difficulties to perform activation measurements in a region too far from the core center.

The detector 2 was used as a monitor as well, and it was positioned in the experimental channel I, approximately in correspondence of the core center. The remaining two detectors were configured as shown in Fig. 5(c). In addition, to fit the holder tube in a channel with larger diameter, apposite ring spacers made out of aluminum were used. The experimental results are shown in Fig. 9, together with a comparison against simulated data. For the Serpent 2 simulation, virtual track-length detector of cylindrical dimension as big as the channel $(54 \mathrm{~mm}$ diameter) are used to characterize the thermal flux profile.

The experimental results show excellent agreement with most of the simulated points, exception made for the data lying in the interval $[20 ; 35] \mathrm{cm}$, where the disagreement reaches values of about $50 \%$. Again, the discrepancy is localized in the zone occupied by the lead shielding, which might have substantial differences in composition with respect to the modeled one.

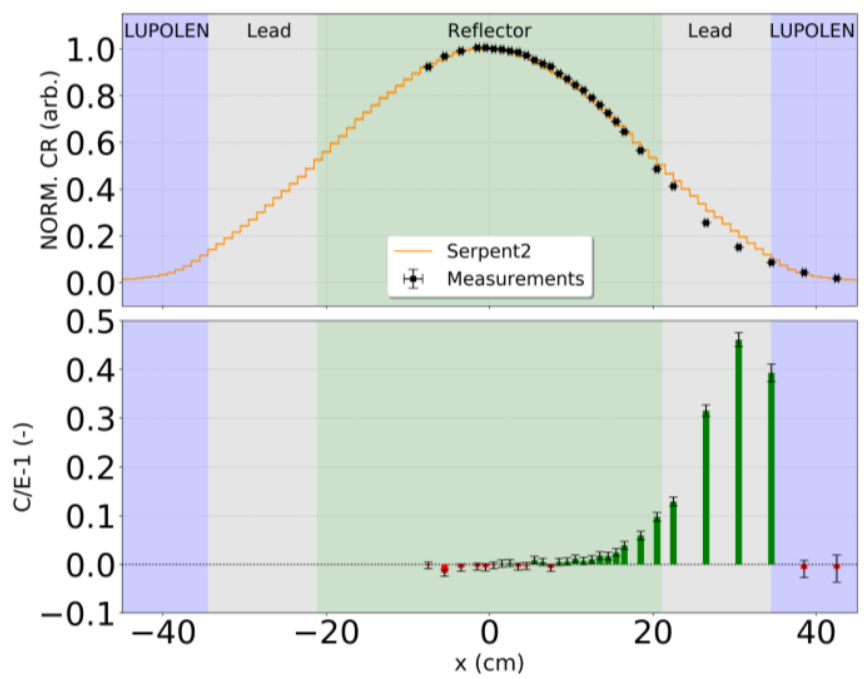

Fig. 9 - Comparison between experimental and simulated results for the thermal neutron flux distribution in tangential channel II.

\section{Thermal neutron flux profiles across channels diameters}

For the experiments on flux profiles across both central and tangential channels, the detectors were positioned in the center of the channel according to the configuration (a) and (c) of Fig. 5 respectively, and then rotated $180^{\circ}$ to repeat the measurement.
Data from detector 2 are used for the central channel, with a power normalization using detector 1 , since its position remains unchanged even after the $180^{\circ}$ rotation. Data from detector 3 are used for the tangential channel, with a power normalization using detector 2 set in the core center.

For the central channel, both experimental and simulation results suggest the absence of a vertical gradient, being the reaction rate distribution almost flat in the core center. However, for the tangential channel II, the experiment shows the existence of a horizontal gradient of $(9.09 \pm 0.20) \%$ from the innermost to the outermost radial position measured with the miniature detectors.

\section{CONCLUSIONS}

High spatial resolution measurements of the thermal neutron flux have been performed with three $1-\mathrm{mm}^{3}$ miniature fibercoupled scintillators within the experimental channels of the SUR-100 facility, a zero-power thermal reactor operated by the IKE of the University of Stuttgart. The miniature detection system was developed at EPFL in collaboration with PSI.

Relative flux distributions were measured along the experimental central (I) and tangential (II) channels of SUR100 and compared against Monte Carlo simulations performed with Serpent 2 . The comparison of experimental and computed neutron distributions showed a good agreement within the core region, with discrepancies within $2 \sigma$. An unexpected discrepancy, probably caused by a geometric inconsistency in the computational model of the reactor, was observed in the reflector region of the experimental channel I, where a $20 \%$ difference (i.e. $8 \sigma$ ) was found between experimental and simulated results. Significant discrepancies, respectively worth $10 \sigma$ and $15 \sigma$, were noticed at distance, in the lead shielding region, for both experimental channels I and II.

The results for the experimental central channel I are compared to neutron activation measurements, showing a strong perturbation of the neutron flux shape (35\% increase of thermal neutron flux in the core region of experimental channel I) due to the plastic-graphite holder used for the samples irradiation. The negligible flux perturbations induced by the miniature fiber-coupled detectors has been found to be a great advantage compared to the previously used manganese activation technique. In addition, reaction rate gradients across the $2.6 \mathrm{~cm}$ and $5.4 \mathrm{~cm}$ diameters of the channels are successfully measured.

An horizontal reaction rate gradient of $(9.09 \pm 0.20) \%$ was measured within $2.4 \mathrm{~cm}$ across the diameter of the experimental channel II, with a difference from computed results of $2 \%$. The absence of a vertical reaction rate gradient inside the experimental channel I was confirmed by measurements.

In conclusion, the experimental campaign carried out in the SUR-100 zero-power reactor highlighted the suitability of the miniature fiber-coupled scintillators for high resolution thermal neutron flux distribution measurements and for the 
characterization of highly localized gradients in the range of few $\mathrm{cm}$.

\section{REFERENCES}

[1] F. Vitullo et al., "A mm3 Fiber-Coupled Scintillator for In-Core Thermal Neutron Detection in CROCUS," IEEE Trans. Nucl. Sci., vol. 67, no. 4, pp. 625-635, Apr. 2020, doi:

10.1109/TNS.2020.2977530.

[2] C. J. Werner et al., "MCNP Version 6.2 Release Notes."

[3] J. Leppänen, M. Pusa, T. Viitanen, V. Valtavirta, and T. Kaltiaisenaho, "The Serpent Monte Carlo code: Status, development and applications in 2013," Ann. Nucl. Energy, vol. 82, pp. 142-150, Aug. 2015, doi: 10.1016/J.ANUCENE.2014.08.024.

[4] F. Vitullo et al., "Developing and testing a miniature fiber-coupled scintillator for in-core neutron counting in CROCUS," Adv. Nucl. Instrum. Meas. Methods Their Appl. (Animma 2019), vol. 225, p. 04018, Jan. 2020, doi: 10.1051/EPJCONF/202022504018.

[5] SCINTACOR, "SCINTACOR - Neutron Screens." [Online]. Available: www.scintacor.com. [Accessed: 29-Jul-2021].

[6] ESKA, "High-Performance Plastic Optical Fiber Specification Sheet SH-8001."

[7] H. Von Gunter and E. Schwartz, "Siemens-Unterrichts-Reaktor SUR Part 2: Reactor description," 1963.

[8] R. J. McConn, C. J. Gesh, R. T. Pagh, R. A. Rucker, and R. Williams III, "Compendium of Material Composition Data for Radiation Transport Modeling," Mar. 2011, doi: 10.2172/1023125. 